\title{
Corrigendum: Biased biological functions of horizontally transfered genes in prokaryotic genomes
}

\author{
Y Nakamura, T Itoh, H Matsuda \& T Gojobori \\ Nat. Genet. 36, 760-766 (2004).
}

NMB0066 of Neisseria meningitidis (Fig. 1b) was the gene artificially introduced within the naturally occurring horizontal transfer cluster candidate in the genome, so that the origin of this gene should be different from that of the surrounding genes.

\section{Corrigendum: Abnormal cerebellar development and axonal decussation due to mutations in AHII in Joubert syndrome}

R J Ferland, W Eyaid, R V Collura, L D Tully, R S Hill, D Al-Nouri, A Al-Rumayyan, M Topcu, G Gascon, A Bodell, Y Y Shugart, M Ruvolo \& C A Walsh

Nat. Genet. 36, 1008-1013 (2004).

The first paragraph of the paper should read "Joubert syndrome is an autosomal recessive brain disorder. Affected individuals show weakness, abnormal breathing and eye movements, clumsiness, cognitive difficulties, and autistic behaviors ${ }^{5-7,10-13}$. Joubert syndrome is defined by the absence of the cerebellar vermis (compare normal MRI, Fig. 1a,b, with the MRI from an individual with Joubert syndrome, Fig. 1d,e) and by the 'molar tooth sign', formed by an abnormal configuration of the superior cerebellar peduncles (SCPs) that connect the cerebellum to the midbrain and thalamus (compare Fig. 1c with Fig. 1f) ${ }^{1-3,5-7,9-12}$. Pathological analysis of Joubert syndrome has shown that this abnormal brainstem structure reflects abnormalities of axons that normally cross the midline, known as commisural fibers. For instance, the SCPs normally cross the midline; failure of the normal crossing causes the molar tooth sign (Fig. 1f $)^{1-3,5-7,9-12}$. Corticospinal fibers...”.

In addition, the legend to Figure 1 was incorrect and should read as follows:

Figure $1 \mathrm{MRIs}$ of a normal brain and of the brain of an individual with Joubert syndrome and mutations in AHI1. MRIs are presented in a coronal plane (a,d, showing the cerebellum and posterior cerebral cortex), in a sagittal plane (b,e, showing a midsagittal section through the brain showing the cerebral cortex, cerebellum, midbrain and pons) and in an axial plane (c,f, showing the upper pons). Coronal (a), midsagittal (b) and axial (c) T1-weighted images from an unaffected child with a normal brainstem $(\mathbf{a}, \mathbf{c})$ and a normal cerebellar vermis $(\mathbf{a}-\mathbf{c})$. Coronal FLAIR (d), midsagittal T1-weighted (e) and axial T1-weighted (f) images from a 2-y-old child with Joubert syndrome and mutations in AHII from pedigree 3. The image shows the molar tooth sign (black arrowhead in $\mathbf{f}$ ) and the absence of the cerebellar vermis (white arrows in $\mathbf{d}, \mathbf{e}$ ). In the axial images from the affected individual (f), the failure of the SCPs to decussate is indicated by the presence of the peduncles traveling horizontal to the brainstem and not crossing the midline. The brain structure that is present in the midsagittal section (e) at the level of the cerebellum is not the vermis but the right lobe of the cerebellum, which is in the plane of the midsagittal section because of the absence of the vermis. Scale bars, $5 \mathrm{~cm}$.

\section{Corrigendum: Evidence for a fruit fly hemangioblast and similarities} between lymph-gland hematopoiesis in fruit fly and mammal aorta-gonadalmesonephros mesoderm

\author{
L Mandal, U Banerjee \& V Hartenstein \\ Nat. Genet. 36, 1019-1023 (2004).
}

The authors wish to acknowledge that the work was supported by grants from the National Institutes of Health (to U.B.) and the National Science Foundation (to V.H.). 